'now'. This suppression mechanism, they propose, is lost in confabulators. The authors relate their own findings to phenomena described in monkeys with orbital frontal lesions, who fail to suppress habits that no longer bring any rewards 9 . Such a mechanism would presumably be part of an error correction process.

This is an interesting proposal, but there are several lines of evidence to suggest that loss of a suppression mechanism is only a partial explanation for confabulations. First, there is the richness of confabulations, exemplified by a patient of ours who described walking down the street the previous day talking to a previous Prime Minister about a joint building job $^{6}$. Second, an individual patient can show confabulations that are stable across time and highly selective in content; one such example is a patient whose only confabulation was a belief that he had to go stock-taking, leading him to dress in formal clothes every morning ${ }^{10}$. It is difficult to see how such bizarre beliefs could arise simply by a general failure to suppress irrelevant associations. A more direct test of the hypothesis may be possible using functional brain imaging. If memory recall requires the suppression of plausible but irrelevant candidate items, then functional imaging should reveal greater activation in the regions Schnider and Ptak specify when a memory retrieval task requires this process than when it does not. The most direct test known to me did not reveal this predicted medial frontal activation ${ }^{11}$, but this may simply reflect the difficulty of imaging this region using $\mathrm{fMRI}^{12}$. Despite these complications, the strength of the new findings and their similarity to the results of habit suppression studies in monkeys will surely provide a motive for further exploration of this interesting question.

1. Kapur, N. \& Coughlan, A. K. J. Neurol.
Neurosurg. Psychiatry 43, 461-463 (1980).

2. Delbecq-Derouesne, J., Beauvois, M. F. \&
Shallice, T. Brain 113, 1045-1074 (1990).

3. Moscovitch, M. in Varieties of Memory and Consciousness: Essays in Honour of Endel Tulving (eds Roediger, H. L. \& Craik, F. I. M.) 133-160 (Erlbaum, Hillsdale, New Jersey, 1989).

4. Damasio, A. R., Graff-Radford, N. R., Eslinger, P. J., Damasio, H. \& Kassell, N. Arch. Neurol. 42, 252-259 (1985).

5. Mercer, B., Wapner, W., Gardner, H. \& Benson, D. Arch. Neurol. 34, 429-433 (1977).

6. Burgess, P. W. \& Shallice, T. Memory 4, 359-411 (1996).

7. Norman, K.A. \& Schacter, D. L. in Implicit Memory and Metacognition (ed. Reder, L. M.) 229-259 (Erlbaum, Hillsdale, New Jersey, 1996).

8. Mesulam, M. M. et al. J. Comp. Neurol. 214, 170-197 (1983).

9. Meunier, M. Bachevalier, J. \& Mishkin, M. Neuropsychologia 35, 999-1015 (1997).

10. Burgess, P. W. \& McNeil, J. E. Cortex 35, 163-182 (1999).

11. Henson, R. N. A., Shallice, T. \& Dolan, R. J. Brain (in press).

12. Frackowiak, R. S. J., Friston, K. J., Frith, C. D., Dolan, R. J. \& Mazziotta, J. C. Human Brain Function (Academic, San Diego, 1997).

\title{
Singing in the brain: song learning in adult zebra finches
}

Many types of synaptic and behavioral plasticity decline as animals mature, but we still do not understand what causes adult behaviors to stabilize. Recently in Nature (399, 466-470, 1999), Anthony Leonardo and Mark Konishi of Caltech reported that zebra finches retain a surprising ability to modify their songs in response to auditory experience in adulthood. Juvenile male zebra finches normally learn to sing by memorizing the song of an older male, and then using auditory feedback to gradually match their own vocalizations to this 'internal model'. Under normal conditions, mature song production then remains stable or 'crystallized' throughout adult life, as shown in the top panel. This stability has been proposed to require active maintenance by auditory feedback, as song gradually deteriorates after deafening in adult birds.

Leonardo and Konishi distorted auditory feedback reversibly, by superimposing timedelayed playback onto the songs produced by adult birds. Within about six weeks, four of five birds had developed substantial song variability, including stuttering, creation, deletion and
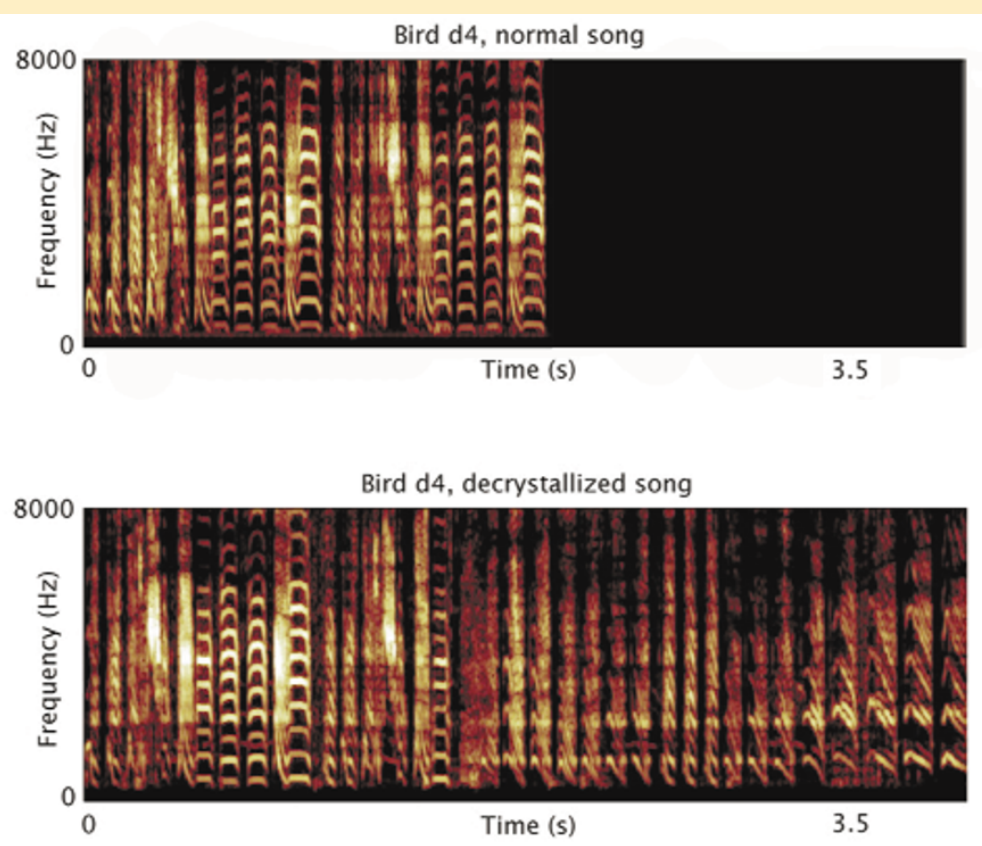
distortion of song elements, as shown in the bottom panel. When the distorted feedback was removed, the birds' songs gradually returned to their stable adult structure, suggesting that the internal model was unaffected by this manipulation. Thus, adult zebra finches retain the ability to adjust their vocal output, but differ from juveniles in that they do not seem to change their internal song model based on auditory input. These findings suggest that stable adult song is maintained by agreement between ongoing auditory feedback and the internal model, rather than because adults lack the ability to modify their song behavior. 